drawings and diagrams to supplement the text but more would have been helpful. The book is divided into fifteen chapters covering most of the basics in clinical ophthalmology including practical chapters on clinical assessment and the management of surgical in-patients which will be much appreciated by busy ophthalmic SHOs. The chapter on ocular motility and strabismus concentrates almost exclusively on paralytic squints and one has to look in the chapter on paediatrics for details of non-paralytic squints, binocular single vision and the cover test. The chapter on paediatric ophthalmology also deals with the visual assessment of children and with the child with poor vision and these aspects are useful. However, many subjects in this chapter such as electro-diagnostic tests, ultrasound, cellulitis and glaucoma are also covered elsewhere in the book whereas childhood uveitis is omitted altogether. There is only sparse coverage of cataract and the ocular effects of systemic diseases and these areas would seem to be particularly relevant to ophthalmic junior staff. Nevertheless these drawbacks are only minor and it must be said that Nicholas Evans has written a good book which will be particularly useful to new entrants to hospital ophthalmology and is to be recommended.

Robert F. Waltern

\section{Eye Diseases in Hot Climates}

By. John Sandford-Smith

Second Edition

Wright: London Boston Singapore Sydney, Toronto, Wellington.

This book is written as a guide to those intending to practice ophthalmology in tropical countries-both the non-specialist already there and the specialist unfamiliar with the tropics. It succeeds on both counts and it is very pleasing to see it in its second edition. The sections on trachoma and onchocerciasis have been updated and the quality of the illustrations is excellent.

There are a few points that could be stressed-the increased safety of using a dry preparation of fluorescein rather than drops for corneal staining the adviseability of giving prophylactic Vitamin A to all children with measles, the use of suturing as a safer procedure than strapping for protecting the cornea, are examples.
Nevertheless this is an extremely useful book with the added advantages of being both easy to read and easily portable and I have no hesitation in recommending it to those working in developing countries.

Marion C. Handscombe

\section{Manual of Visual Fields}

Elliott B. Werner

Churchill Livingstone

This is a concise book covering visual field assessment and diagnosis and the modern instrumentation available. The fundamentals of field technique using the Goldmann Perimeter are well discussed though much of the book relates to autoperimetry using the Octopus and Humphrey Field Analysers.

In the historical review of field testing, campimetry technique using the Bjerrum Tangent Screen is rather glossed over, even though its continuing usefulness is referred to later in the text, especially in the context of functional disease. There is passing reference only to the Tubinger instrument, and slight reference to the computer of Heijl, and the screening devices of Freidman, and Hansen, as also the computer display method of Damato.

Nevertheless the book gives an excellent description of the principles of dynamic and static field testing using threshold and supratheshold determinants, and the handling of the physical units involved is well presented. In this respect the concept of differential retinal sensitivity and threshold are logically and carefully expounded in a clear and readable manner. The comparative methods and testing logic of the three important perimeters (Goldmann, Octopus, and Humphrey) are nicely collected here in one book which allows easy cross reference of their equivalence.

The illustrations and diagrams are all derived from clinical material and are excellent. The statistical analysis software (Octosoft, Statpac Humphrey), are well explained.

In the clinical section there are chapters on field defects with media opacities, retinal and choroidal lesions, glaucoma, optic nerve, chiasm, and retrochiasmal pathways. There are clear diagnostic criteria as well as useful hints. The pitfalls of autoperimetry are also indicated. 
This book is of value to the practicing clinician, to post graduate students and to fields technicians alike. It is a worthy addition to their working library.

Greg Munton

\section{Ophthalmology Monographs 1 Retinal Detachment}

George F. Hilton, Edward B. McLean, Elaine L. Chaung

American Academy of Ophthalmology

This short book is produced by the American Academy of Ophthalmology and is intended for residents in training.

It is attractively produced, with good colour photographs and references at the end of each chapter are generally well chosen.

The quality of the chapters reflects its multiauthorship. The introductory chapters on pathogenesis and natural history of retinal detachment are of good quality and appropriate complexity for the beginner.

The chapter concerning preoperative management for retinal reattachment surgery is poor. One surgeon's practise is described, with no reference to studies which might support his recommended management-for example that patients should routinely be given laxatives daily and chloral hydrate sedation at night.

The section on retinopexy opens with a discussion of the use of scleral diathermy-a method long since discarded to the pages of the history books by most retinal surgeons. A discussion of scleral buckling describes in detail techniques for scleral resection and oversewing whilst too little space is given to the use of scleral explants.

The book claims that a discussion of vitrectomy is beyond its scope. It shouldn't be. The ability to identify cases where primary vitrectomy is appropriate is an essential part of the training of all ophthalmologists and its exclusion from this monograph is wrong.

Despite the good quality of the production of this volume and the excellent opening chapters, the faults in the sections concerning surgical management are such that this monograph cannot be recommended to ophthalmic surgeons in training.

Wendy Franks

\section{Radiology of the Eye and Orbit}

T. H. Newton, L. T. Bilaniuk, eds

(Modern Neuroradiology, Volume 4).

A Clavadel Book. pp x +350 . Raven Press, New York 1990.

This substantial addition to the literature on ocular and orbital imaging covers magnetic resonance imaging (MRI), ultrasonography and X-ray computed tomography (CT). For most ophthalmologists the technical chapters on ultrasonography and MRI are probably of very restricted interest, and those on anatomy also of limited appeal. The meat of the book consists of about 200 profusely illustrated pages on imaging of pathology of the eye and orbit. However, many ophthalmologists referring patients for imaging are subspecialists and for those beyond the training stage, I am not sure that orbital imaging will greatly concern those who frequently use ocular ultrasound, and vice versa.

The quality of the CT and ultrasound studies is generally excellent. That of the MR images, particularly of some of the rarer conditions, is understandably more variable. Unfortunately, at some points text and relevant illustrations are ten or more pages apart.

British readers may find themselves at loggerheads with some of the views explicit or implicit in the text, such as disparagement of STIR imaging of the optic nerve, and loyalty to the concept of orbital lymphangiomas. The CT chapters in particular were written some years ago (and are reprinted from Volume 3 of this series), so some conditions discussed in more recent journal articles, such as retrobulbar neuritis, orbital liposarcoma or intraconal haematic cyst, are rather neglected.

I believe strongly that the skeleton for radiological textbooks, especially those aimed at clinicians, should be clinical presentations or at least types of pathology, but this, like most, is organised by techniques; an additional chapter, critically evaluating the utility in clinical management of the methods described, would have made this book more valuable for the ophthalmologist, and probably for the imaging specialist too.

Ivan Moseley 